\title{
DETERMINAÇÃO DE ETANOL E VOLÁTEIS RELACIONADOS EM SANGUE E FLUIDO ORAL POR MICROEXTRAÇÃO EM FASE SÓLIDA EM HEADSPACE ASSOCIADA À CROMATOGRAFIA GASOSA COM DETECTOR DE IONIZAÇÃO EM CHAMA
}

\author{
Lílian de Lima Feltraco, Marina Venzon Antunes e Rafael Linden* \\ Instituto de Ciências da Saúde, Centro Universitário Feevale, Rodovia RS 239, n. 2755, 93352-000 Novo Hamburgo - RS, Brasil
}

Recebido em 2/2/09; aceito em 11/5/09; publicado na web em 6/10/09

\begin{abstract}
DETERMINATION OF ETHANOL AND RELATED VOLATILE COMPOUNDS IN BLOOD AND ORAL FLUID BY HEADSPACE SOLID-PHASE MICRO EXTRACTION ASSOCIATED TO GAS CHROMATOGRAPHY WITH FLAMEIONIZATION DETECTOR. The aim of this study was to validate a method for the determination of acethaldehyde, methanol, ethanol, acetone and isopropanol employing solid-phase microextraction associated to gas chromatography with flame ionization detection. The operational conditions of SPME were optimized by response surface analysis. The calibration curves for all compounds were linear with $\mathrm{r}^{2}>0.9973$. Accuracy $(89.1-109.0 \%)$, intra-assay precision (1.8-8.5\%) and inter-assay precision $(2.2-8.2 \%)$ were acceptable. The quantification limit was $50 \mu \mathrm{g} / \mathrm{mL}$. The method was applied to the meaurement of ethanol in blood and oral fluid of a group of volunteers. Oral fluid ethanol concentrations were not directly correlated with blood concentrations.
\end{abstract}

Keywords: ethanol; SPME-GC-FID; oral fluid.

\section{INTRODUÇÃO}

A determinação de etanol em sangue é uma das análises mais frequentes e importantes realizadas pelos laboratórios de toxicologia clínica e forense, com nível máximo aceitável em sangue de $0,2 \mathrm{~g} / \mathrm{L}$, determinado pela legislação brasileira para a condução de veículos. ${ }^{1}$ Além do etanol, outros compostos voláteis de relevância toxicológica também devem ser determinados em amostras biológicas. Os álcoois metanol e isopropanol também são depressores do sistema nervoso central, com níveis tóxicos inferiores ao do etanol, responsáveis por diversas intoxicações fatais. ${ }^{2} \mathrm{~A}$ acetona pode estar presente em concentrações elevadas nos quadros de cetoacidose diabética, cujo quadro clínico é semelhante à intoxicação alcoólica, sendo sua determinação importante para o diagnóstico diferencial. ${ }^{3}$ O acetaldeído pode ser utilizado como marcador de putrefação das amostras e também é o principal metabólito do etanol. ${ }^{3}$ Em 2002, Zuba e colaboradores demonstraram a presença de níveis mensuráveis de acetaldeído, metanol, acetona e isopropranol em amostras de sangue de alcoolistas crônicos. ${ }^{4}$

Embora o sangue seja a matriz preferencial para a avaliação da exposição aos compostos voláteis acima mencionados, diversos trabalhos têm discutido a aplicação de amostras de fluido oral para esta determinação. A utilização de amostras de fluido oral apresenta como vantagem a possibilidade de uma coleta assistida e não invasiva, mesmo em condições de campo. Para a obtenção de amostras de fluido oral são utilizados vários dispositivos comerciais, conforme revisado por Crouch. ${ }^{5}$ Recentemente um novo sistema para coleta foi introduzido pela empresa Greiner Bio-One, o qual permite a lavagem prévia da cavidade oral e a determinação exata da quantidade de saliva recuperada através de medidas espectrofotométricas. Até o momento não foram publicados trabalhos com a utilização deste sistema para obtenção de amostras para a determinação de etanol e outros compostos voláteis.

A forma mais usual para a determinação de etanol e compostos relacionados em fluidos biológicos é pela cromatografia gasosa com amostragem por headspace estático associada a detectores de ioniza-

*e-mail: rafael.linden@ feevale.br ção em chama, descrita nos anos 70 do século XX. ${ }^{6}$ Uma alternativa mais recente é o emprego de microextração em fase sólida (MEFS) para amostragem e pré-concentração dos analitos, que apresenta como vantagens a inexigibilidade do uso de solventes orgânicos e a possibilidade de automação. A MEFS já foi aplicada para a determinação de etanol em procedimento manual, ${ }^{7}$ assim como com a utilização de sistemas automatizados. ${ }^{8,9}$ Cabe destacar que a determinação das condições experimentais de máxima eficiência da MEFS é um problema multifatorial, sendo influenciada por condições experimentais como a temperatura das amostras, tempo de exposição da fibra e quantidade de agente de salting out utilizado. Uma forma produtiva e eficiente para determinar as condições experimentais otimizadas para a MEFS, empregada neste estudo, é a utilização de experimentos fatoriais, os quais permitem calcular condições otimizadas a partir de um número limitado de experimentos adequadamente planejados. ${ }^{10}$

Desta forma, o objetivo do presente trabalho foi desenvolver e validar um método para a determinação simultânea de acetaldeído, metanol, etanol, acetona e isopropanol empregando MEFS automatizada associada à cromatografia gasosa com detector de ionização em chama (MEFS-CG-DIC) e aplicação à determinação destes compostos em amostras de sangue e fluido oral em um estudo cinético de etanol com um grupo de voluntários.

\section{PARTE EXPERIMENTAL}

\section{Reagentes e materiais}

Metanol, etanol e acetona foram provenientes da J. T. Baker(Cidade do México, México), acetaldeído foi proveniente Acros Organics (New Jersey, EUA) e n-propanol e isopropanol foram provenientes da Merck (Darmstadt, Alemanha). O cloreto de sódio foi obtido da Nuclear (São Paulo, Brasil). A água utilizada foi purificada através de um sistema Elga Purelab Ultra da Elga Labwater (Lane End, Reino Unido). A fibra para MEFS revestida de poliacrilato (PA), com espessura do filme de $85 \mu \mathrm{m}$, foi fornecida pela Supelco (Bellefonte, Estados Unidos). A fibra foi condicionada através da sua inserção no injetor do cromatógrafo aquecido a $250{ }^{\circ} \mathrm{C}$, sendo mantida nesta condição por $30 \mathrm{~min}$, conforme instruções do fabricante. 


\section{Equipamentos}

Todo o sistema de cromatografia gasosa foi proveniente da Varian (Midlesburg, Holanda). O procedimento de MEFS foi realizado em um autoamostrador Varian CP-8410, com bandeja para recirculação de água. A temperatura das amostras foi controlada através de banhomaria com circulação de água Nova Ética (Vargem Grande Paulista, Brasil). As análises foram realizadas em cromatógrafo a gás Varian CP-3800, com detector de ionização em chama. O sistema cromatográfico foi controlado pelo programa Galaxie. A coluna capilar utilizada na separação cromatográfica foi uma RTX-BAC 2 fornecida pela Restek (Bellefonte, Estados Unidos), com $30 \mathrm{~m}$ de comprimento; $0,53 \mathrm{~mm}$ de d.i. e revestimento interno com $2 \mu \mathrm{m}$ de espessura.

\section{Condições cromatográficas}

A temperatura do injetor, operado na condição sem divisão de fluxo, foi de $200^{\circ} \mathrm{C}$. Durante as análises a coluna foi mantida a $40{ }^{\circ} \mathrm{C}$, com duração de corrida cromatográfica de $7 \mathrm{~min}$. Hélio ultrapuro foi utilizado como gás de arraste, com fluxo constante de $12,5 \mathrm{~mL} / \mathrm{min}$. Foi empregado um detector de ionização em chama, mantido a $200^{\circ} \mathrm{C}$.

\section{Otimização da MEFS}

As condições experimentais para a MEFS foram selecionadas através de Metodologia de Superfície de Resposta, que é definida como um conjunto de técnicas matemáticas e estatísticas empregadas para modelar e analisar problemas nos quais uma variável de resposta é influenciada por diversas variáveis operacionais com o objetivo de otimizar esta resposta. ${ }^{11}$ Através de um desenho experimental adequado é possível obter uma equação de regressão de segunda ordem que descreve a relação entre as variáveis do processo e a variável de resposta. Foi selecionado um desenho experimental do tipo Box-Behnken, com as seguintes variáveis experimentais: temperatura das amostras $\left(40,50\right.$ e $\left.60{ }^{\circ} \mathrm{C}\right)$, massa de cloreto de sódio na amostra $(0,05 ; 0,13$ e $0,20 \mathrm{~g})$ e tempo de exposição da fibra durante a etapa de adsorção (5, 10 e 15 min), em um total de 17 experimentos. Para determinação das condições experimentais otimizadas foi utilizada a Função de Preferência, na qual cada resposta é representada por uma função individual de preferência, com valores entre 0 e 1 , conforme Derringer e Suich. ${ }^{11}$ Quando a resposta está em seu valor desejado, a função de preferência assume o valor 1 . Por outro lado, quando a resposta está fora de uma região aceitável, o valor da função é 0 . O valor final de Preferência foi determinado através da média geométrica das funções individuais de Preferência para cada um dos analitos. ${ }^{10}$ Neste experimento, foram calculadas soluções com o objetivo de maximizar a área dos picos de todos os analitos. Os cálculos foram realizados utilizando o programa Design Expert $^{\circledast}$ da Stat-Ease (Minneapolis, EUA).

\section{Preparo das amostras}

A preparação das amostras pela técnica de MEFS por headspace seguiu as condições determinadas após o experimento de otimização. Em tubo de ensaio com tampa de rosca, contendo cloreto de sódio, foram adicionados $100 \mu \mathrm{L}$ de amostra, $50 \mu \mathrm{L}$ da solução de trabalho de padrão interno (n-propanol $1.870 \mu \mathrm{g} / \mathrm{mL}$ ) e $400 \mu \mathrm{L}$ de água purificada. No processamento das amostras de fluido oral, foi utilizado um volume de água purificada suficiente para que o volume total da solução de coleta de fluido oral adicionada à água purificada fosse de $500 \mu \mathrm{L}$. A mistura foi homogeneizada em vortex por $1 \mathrm{~min}$ e uma alíquota de $500 \mu \mathrm{L}$ foi transferida para um frasco com $2 \mathrm{~mL}$ de capacidade com septo de teflon e colocada em autoamostrador com temperatura controlada, com exposição do headspace à fibra de MEFS durante o tempo otimizado. A dessorção foi realizada por exposição da fibra ao injetor do cromatógrafo por $2 \mathrm{~min}$.

\section{Avaliação da seletividade}

Amostras de sangue e fluido oral de 6 indivíduos não expostos aos analitos foram processadas e a presença de picos interferentes nos tempos de retenção dos analitos foi avaliada.

\section{Curva de calibração}

Para a construção da curva de calibração foi utilizada a média de três determinações de cada calibrador contendo acetaldeído, metanol, etanol, acetona e isopropanol nas concentrações de 50, 100, 200, $500,2.000,1.000$ e $5.000 \mu \mathrm{g} / \mathrm{mL}$. Os calibradores foram preparados através da diluição (1:10), com sangue ou fluido oral brancas, de soluções aquosas contendo os analitos nas concentrações 500, 1.000, $2.000,5.000,10.000,20.000$ e $50.000 \mu \mathrm{g} / \mathrm{mL}$. A curva de calibração foi construída através de regressão linear pelo método dos mínimos quadrados, estabelecendo a correlação entre a razão da área dos picos referentes dos analitos e a área do pico de n-propanol (y) e as concentrações adicionadas (x). A curva foi avaliada com base no coeficiente de determinação $\left(\mathrm{r}^{2}\right)$. A equação de regressão foi obtida empregando o programa Microsoft Excel ${ }^{\circledR}$.

\section{Avaliação do efeito de memória da fibra de MEFS}

Foram processadas amostras de sangue e fluido oral contendo todos os analitos na concentração de $5.000 \mathrm{mg} / \mathrm{mL}$, sucedidas de amostras brancas, em triplicata. O efeito de memória foi avaliado através da determinação da área dos picos de acetaldeído, metanol, etanol, acetona e isopropanol nas amostras brancas.

\section{Ensaios de precisão e exatidão}

A precisão e a exatidão do método foram avaliadas através de análises em três níveis de concentração para cada analito, realizadas em triplicata e repetidas em 5 dias diferentes. Os calibradores utilizados foram preparados em sangue e fluido oral isentos dos analitos. A precisão intraensaios e a precisão interensaios foram calculadas pela análise de variância (ANOVA) usando dia como variável agrupadora, seguindo o modelo experimental de Wieling et al. ${ }^{12}$ A exatidão foi calculada como percentagem média obtida do valor teórico adicionado na amostra. As concentrações utilizadas no estudo de precisão e exatidão foram 100, 400 e $4.000 \mu \mathrm{g} / \mathrm{mL}$.

\section{Estabilidade}

Amostras de sangue e fluido oral adicionadas de acetaldeído, metanol, etanol, acetona e isopropanol na concentração de 1.000 $\mathrm{mg} / \mathrm{mL}$ foram preparadas e imediatamente armazenadas a $4{ }^{\circ} \mathrm{C}$, em tubos de ensaio de vidro com tampa rosca. Alíquotas destas amostras foram analisadas no dia da preparação e 14 dias após. A estabilidade foi avaliada através da diferença percentual entre o valor determinado no início e no final da série.

\section{Limite de quantificação}

O limite de quantificação para cada analito foi definido como a menor concentração de um calibrador apresentando coeficiente de variação percentual (C.V. \%) intraensaios inferior a $20 \% .^{13}$ 


\section{Estudo com voluntários}

Um grupo de 6 voluntários recebeu uma dose oral de etanol, na forma de bebida destilada (uísque), fornecendo amostras de sangue e fluido oral nos tempos 0,$5 ; 1,2$ e 3 h após a administração. Também foi coletada uma amostra imediatamente antes da administração de etanol, a fim de eliminar do estudo voluntários com níveis basais de etanol. Os voluntários foram orientados a não consumir alimentos 2 $\mathrm{h}$ antes da administração da bebida. Considerando as diferenças no volume de distribuição entre homens e mulheres, a dose foi de 30 $\mathrm{g}$ de etanol para os voluntários do sexo masculino $(\mathrm{n}=2)$ e $40 \mathrm{~g}$ de etanol para os voluntários do sexo feminino $(n=4)$. As doses foram ingeridas em um tempo máximo de $10 \mathrm{~min}$

As amostras de sangue periférico foram coletadas através de punção venosa para tubos contendo EDTA como anticoagulante, a vácuo, em um volume máximo de $4 \mathrm{~mL}$. As amostras de fluido oral foram obtidas conforme o procedimento descrito no ítem "obtenção das amostras de fluido oral". Imediatamente após a coleta, as amostras foram armazenadas sob refrigeração a $4{ }^{\circ} \mathrm{C}$. As análises foram realizadas no mesmo dia da coleta.

No dia anterior ao estudo, os voluntários forneceram uma amostra de sangue venoso a fim de permitir a determinação dos níveis de creatinina e transaminases séricas. Alterações nestes parâmetros, existência de patologias, uso de medicamentos e histórico de abuso de substâncias foram critérios de exclusão do estudo. O protocolo empregado neste estudo foi aprovado pelo Comitê de Ética em Pesquisa do Centro Universitário Feevale e todos os voluntários assinaram um termo de consentimento.

\section{Obtenção das amostras de fluido oral}

As amostras de fluido oral foram obtidas através do Saliva Collection System, fornecido gentilmente pela Greiner Bio-One (Kremsmünster, Áustria). Este sistema é composto de 4 frascos, a saber: frasco com solução de lavagem, frasco com solução de coleta contendo ácido cítrico e corante, frasco para coleta de fluido oral e frasco para armazenamento de amostra. Uma característica deste sistema de coleta é a presença do corante alimentício amarelo tartrazina na solução de coleta, que permite a determinação quantitativa da proporção de fluido oral recuperado durante o procedimento. $\mathrm{O}$ procedimento de coleta do fluido oral inicia com a lavagem da cavidade oral com a solução de lavagem (frasco 1), a qual deve ser bochechada por pelo menos $30 \mathrm{~s}$. Após, a cavidade oral é enxaguada com $4 \mathrm{~mL}$ da solução de coleta (frasco 2) por 2 min, com a transferência da mistura obtida para o frasco de coleta (frasco 3). O conteúdo do frasco de coleta é transferido para os tubos de armazenamento (frasco 4), que contêm o preservante azida de sódio, por sucção a vácuo. Após a finalização do procedimento, os tubos de armazenamento são centrifugados por $10 \mathrm{~min}$ a $2.000 \mathrm{~g}$.

\section{Determinação da proporção de fluido oral na solução de coleta}

A proporção de fluido oral na solução de coleta foi determinada através do Saliva Quantification Kit, fornecido gentilmente pela Greiner Bio-One (Kremsmünster, Áustria). Este kit é composto de calibradores contendo uma mistura da solução de coleta de saliva e de fluido oral, o qual está presente nas proporções 11, 27, 46, 66 e $85 \%$. A diferença entre as absorbâncias medidas em 450 e $520 \mathrm{~nm}$ foi determinada para cada um dos calibradores e uma regressão linear descrevendo a relação entre estes valores (y) e a proporção de fluido oral (x) foi calculada. A equação obtida foi $y=-0,0184 x+1,8528$, com $\mathrm{r}^{2}$ de 0,9999. Para cada amostra de fluido oral coletada, após a obtenção da diferença de absorbância, foi calculada a proporção de fluido oral contida no tubo de armazenamento. A quantidade de solução equivalente a $100 \mu \mathrm{L}$ de fluido oral foi utilizada no procedimento de quantificação de etanol e demais analitos.

\section{RESULTADOS E DISCUSSÃO}

\section{MEFS}

A técnica de MEFS apresenta como principais vantagens a inexigibilidade de solventes orgânicos e a possibilidade de automação, o que pode contribuir para obtenção de métodos analíticos com maior segurança para técnicos e o meio ambiente e com elevada produtividade. Entretanto, a determinação das condições experimentais mais adequadas se apresenta como um problema multifatorial, que pode ser abordado por estratégias de tentativa e erro ou, alternativamente, através de experimentos estatisticamente planejados. A segunda abordagem em geral é mais eficiente, minimizando o número de experimentos, e permite modelar matematicamente os processos estudados, permitindo o cálculo de condições otimizadas. Neste estudo, a otimização da MEFS foi realizada através de um desenho experimental do tipo Box-Behnken devido a sua economicidade e pela possibilidade de modelar superfícies de resposta. As variáveis estudadas foram: concentração do agente de salting out (cloreto de sódio), temperatura da amostra e tempo de exposição da fibra de MEFS ao headspace da amostra. A variável otimizada foi a área dos picos dos analitos. Os níveis das variáveis estudadas e os resultados obtidos para as áreas dos picos são apresentados na Tabela 1. Os modelos matemáticos obtidos para descrever todas as respostas foram significativos $(\mathrm{P}>0,05)$.

As condições otimizadas foram determinadas através do cálculo de soluções para a Função de Preferência e representadas graficamente através das superfícies de resposta, empregando o programa Design Expert. A solução com maior Preferência $(0,994)$ foi aquela com as três variáveis investigadas em seu nível máximo. Entretanto, considerando que a função de preferência apresenta um platô (Figura 1), com pequena diferença com a redução do tempo de exposição da fibra para $10 \mathrm{~min}$, e a necessidade de otimizar a produtividade laboratorial, as condições operacionais selecionadas para a validação e aplicação do método foram: temperatura da amostra de $60{ }^{\circ} \mathrm{C}$, quantidade de cloreto de sódio de $0,20 \mathrm{~g}$ e tempo de exposição da fibra ao headspace da amostra de $10 \mathrm{~min}$. Esta condição apresentou valor de preferência de 0,970, marginalmente inferior à melhor condição calculada.

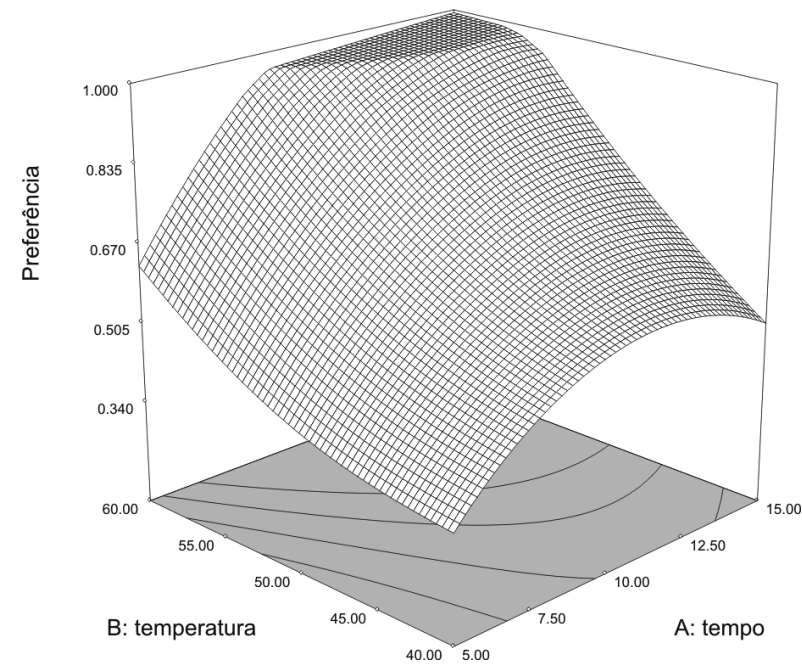

Figura 1. Superfície de resposta da função de preferência em função das variáveis temperatura da amostra e tempo de exposição da fibra. O fator quantidade de $\mathrm{NaCl}$ está fixado em $0,20 \mathrm{~g}$ 
Tabela 1. Desenho experimental de otimização da MEFS

\begin{tabular}{lcccccccc}
\hline Experimento & $\begin{array}{c}\text { Tempo } \\
(\mathrm{min})\end{array}$ & $\begin{array}{c}\text { Temperatura } \\
\left({ }^{\circ} \mathrm{C}\right)\end{array}$ & $\begin{array}{c}\mathrm{NaCl} \\
(\mathrm{g})\end{array}$ & Acetaldeído & Etanol & Metanol & Acetona & Isopropanol \\
\hline 1 & 5,00 & 50,00 & 0,05 & 111,6 & 185,3 & 75,7 & 210,4 & 164,5 \\
2 & 15,00 & 40,00 & 0,13 & 160,2 & 252,0 & 90,6 & 388,2 & 335,2 \\
3 & 15,00 & 60,00 & 0,13 & 224,4 & 381,0 & 140,3 & 589,1 & 616,2 \\
4 & 10,00 & 40,00 & 0,20 & 174,6 & 337,8 & 109,7 & 474,1 & 517,9 \\
5 & 15,00 & 50,00 & 0,05 & 165,4 & 226,7 & 96,3 & 340,4 & 248,1 \\
6 & 10,00 & 40,00 & 0,05 & 174,6 & 337,8 & 109,7 & 474,1 & 517,9 \\
7 & 10,00 & 50,00 & 0,13 & 199,5 & 264,8 & 104,3 & 458,1 & 414,1 \\
8 & 5,00 & 40,00 & 0,13 & 142,9 & 321,1 & 85,4 & 310,8 & 272,5 \\
9 & 10,00 & 50,00 & 0,13 & 195,6 & 279,2 & 110,5 & 480,3 & 450,8 \\
10 & 10,00 & 50,00 & 0,13 & 195,1 & 280,2 & 109,5 & 481,7 & 471,8 \\
11 & 10,00 & 60,00 & 0,05 & 163,7 & 203,2 & 99,4 & 329,0 & 259,0 \\
12 & 10,00 & 60,00 & 0,20 & 238,3 & 403,8 & 141,9 & 611,1 & 702,4 \\
13 & 10,00 & 50,00 & 0,13 & 202,2 & 290,7 & 114,3 & 497,9 & 473,8 \\
14 & 15,00 & 50,00 & 0,20 & 212,9 & 376,3 & 124,3 & 577,7 & 626,4 \\
15 & 10.00 & 50.00 & 0,13 & 190,8 & 278,6 & 110,3 & 464,9 & 445,8 \\
16 & 5.00 & 50.00 & 0,20 & 172,6 & 239,2 & 94,3 & 410,6 & 575,0 \\
17 & 5.00 & 60.00 & 0,13 & 152,4 & 282,8 & 99,0 & 372,4 & 349,4 \\
\hline
\end{tabular}

A automação da MEFS foi realizada através de um autoamostrador CP-8410 modificado através da adaptação de um de banho-maria com circulação de água à bandeja de posicionamento das amostras. Esta modificação permitiu controle de todas as variáveis importantes da MEFS em um sistema de baixo custo, em comparação com os robôs de automação de MEFS disponíveis no mercado.

\section{Condições cromatográficas}

O tempo total da análise cromatográfica foi de $7 \mathrm{~min}$. As condições cromatográficas utilizadas permitiram a separação completa dos analitos estudados. Os tempos de retenção médios foram 1,33 min para acetaldeído; 1,69 min para metanol; 2,21 min para etanol; 2,37 min para acetona; 2,62 min para isopropanol e 4,25 para o padrão interno n-propranol. Embora tinha sido obtida uma separação cromatográfica completa em menos de $5 \mathrm{~min}$, a corrida teve a duração total de $7 \mathrm{~min}$, a fim de permitir a eluição de compostos de maior massa molecular. Cabe destacar que o limitante no tempo de análise foi o tempo de exposição da fibra de MEFS. Considerando o tempo de exposição da fibra de 10 min, o tempo de dessorção no injetor de 2 min e o tempo necessário para a movimentação das partes móveis do autoamostrador foi possível analisar uma amostra a cada $14 \mathrm{~min}$, aproximadamente. A Figura 2 apresenta cromatogramas típicos obtidos pela aplicação do método, nos quais não são observados picos além dos de interesse. Apesar dos picos apresentarem tendência à formação de caudas, o perfil cromatográfico manteve-se inalterado mesmo após 500 injeções, sem comprometimento do desempenho quantitativo do método. As amostras de sangue total e fluido oral apresentam perfil cromatográfico semelhante, com ausência de interferentes nos estudos de seletividade com amostras de voluntários.

As curvas de calibração, apresentadas na Tabela 2, apresentaram adequada linearidade, com coeficientes de determinação $\left(\mathrm{r}^{2}\right)$ superiores a 0,9973 . Os valores quantitativos obtidos não foram influenciados por eventual efeito de memória da fibra de MEFS, visto que amostras de sangue e fluido oral isentas dos analitos não apresentaram picos detectáveis quando processadas imediatamente após amostras com concentrações iguais ao calibrador de maior nível. Considerando a elevada repetibilidade do procedimento de MEFS proporcionado pela automação, foi possível obter determinações quantitativas com precisão e exatidão adequadas, conforme dados obtidos nos experimentos de
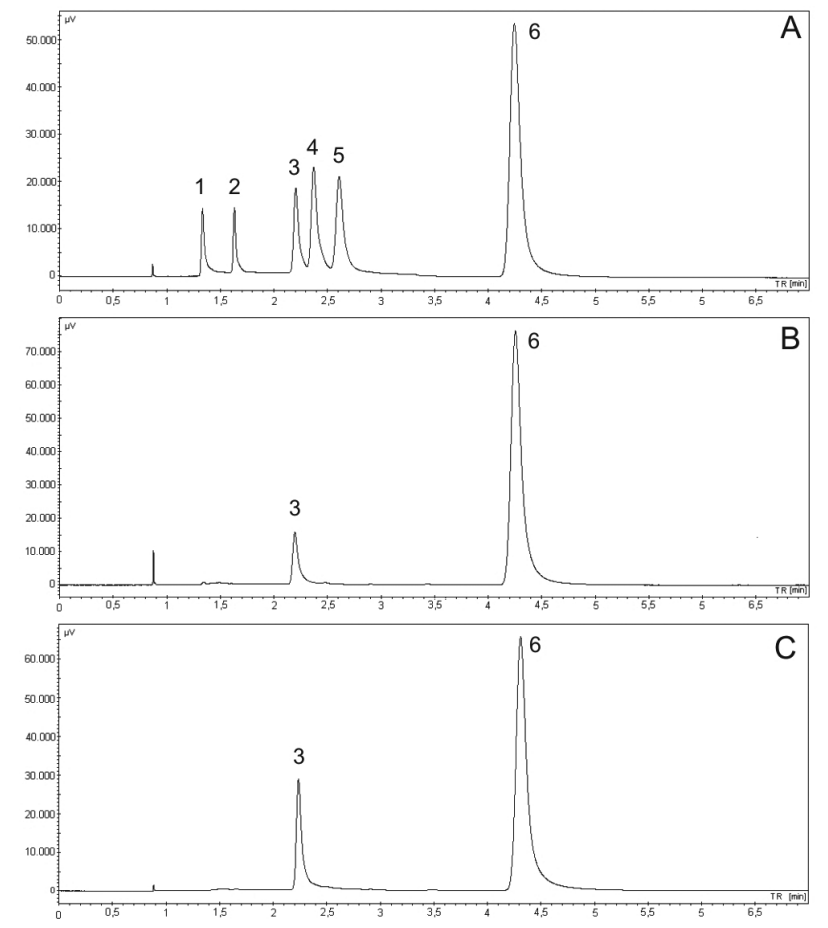

Figura 2. Separação cromatográfica: (A) calibrador preparado em sangue contendo acetaldeído (1), metanol (2), etanol (3), acetona (4), isopropanol (5) e propanol (6), todos a 400,0 $\mu \mathrm{g} / \mathrm{mL}$; (B) amostra de sangue de voluntário obtida $1 \mathrm{~h}$ após a ingesta de etanol (341,2 $\mu \mathrm{g} / \mathrm{mL})$; (C) amostra de fluido oral do mesmo voluntário em $B$, obtida 1 h após a ingesta de etanol $(688,5 \mu \mathrm{g} / \mathrm{mL})$ 
validação e apresentados na Tabela 2 . Os valores obtidos de precisão intraensaios (1,8-8,5\%), precisão interensaios $(2,2-8,2 \%)$ e exatidão (89,1-109,0\%) encontram-se dentro dos critérios de aceitação para métodos bioanalíticos preconizados por Shah et al.. ${ }^{13}$ Não foi observado nenhum pico cromatográfico nos tempos de retenção dos analitos quando amostras brancas foram analisadas imediatamente após amostras de sangue e fluido com todos os analitos em concentração de 5.000 $\mu \mathrm{g} / \mathrm{mL}$, demonstrando a inexistência de efeito de memória na fibra de MEFS. As concentrações dos analitos mostraram-se estáveis quando as amostras de sangue e fluido oral foram mantidas em refrigeração, em um intervalo de até 14 dias, com diferença percentual entre os valores pré e pós-armazenamento de $5,1 \%$.

O limite de quantificação para todos os analitos foi de $50 \mu \mathrm{g} / \mathrm{mL}$. Considerando o propósito do método proposto neste estudo, focado no diagnóstico laboratorial clínico e forense das intoxicações pelos compostos avaliados, os limites de quantificação são significativamente inferiores aos níveis tóxicos disponíveis na literatura para metanol, etanol, acetona e isopropanol (200, 1.000, 200 e $200 \mu \mathrm{g} / \mathrm{mL}$, respectivamente), mas superiores ao relatados para acetaldeído $(20 \mu \mathrm{g} / \mathrm{mL}){ }^{2}$ Além disto, o pequeno volume de amostra utilizado permite a utilização de amostras de volume reduzido, o que normalmente é o caso de amostras de fluido oral.

\section{Estudo com voluntários}

Na Tabela 3 são apresentados os intervalos das concentrações obtidas através das análises das amostras de sangue e fluido oral dos voluntários participantes do estudo, bem como a razão entre as concentrações.

Jones encontrou uma razão média entre concentrações de etanol em fluido oral e sangue, após uma administração oral, de $1,08 \cdot{ }^{14}$ Esta razão foi constante no intervalo de 60 até 360 min após a administração. Em um estudo posterior de Jones, a razão média foi 1,09, com faixa de valores entre 0,88 e 1,36. ${ }^{15}$ Gubala e Zuba encontram diferenças médias entre as concentrações de etanol em sangue e fluido oral que se estabilizaram após 90 min da ingesta de etanol, com concentrações sempre superiores no fluido oral por um fator médio de $1,08 .{ }^{16}$
Em nosso estudo também observamos concentrações de etanol no fluido oral marcadamente superiores nos tempos 0,5 e $1 \mathrm{~h}$, por um fator médio de 2,61 e 1,87, respectivamente. Entretanto, a partir dos tempos de amostragem posteriores as diferenças mostraram uma tendência de redução, apresentando razão média entre as concentrações em fluido oral e sangue de 1,36 no tempo $2 \mathrm{~h}$ e de 1,15 no tempo $3 \mathrm{~h}$. Naturalmente, o pequeno número de amostras avaliadas no estudo, associada à variabilidade das razões de concentrações, limita as conclusões sobre as diferenças encontradas. Entretanto, pode-se inferir que, de acordo com os achados já publicados, as diferenças de concentração de etanol entre fluido oral e sangue apresentam um comportamento relativamente previsível, viabilizando a utilização do fluido oral como amostra diagnóstica somente após algumas horas após a ingesta. Entretanto, não é possível uma estimativa exata das concentrações de etanol no sangue a partir das concentrações obtidas em fluido oral. Também, o Saliva Collection System mostrou-se adequado para a obtenção de amostras de fluido oral, em quantidades adequadas para a análise.

\section{CONCLUSÃO}

Um método para a determinação de etanol, acetaldeído, metanol, acetona e isopropanol em amostras de sangue e fluido oral por headspace-MEFS-CG-DIC foi desenvolvido e validado. O procedimento de MEFS foi otimizado com respeito aos parâmetros temperatura da amostra, quantidade de $\mathrm{NaCl}$ na amostra e tempo de exposição da fibra empregando metodologia de superfície de resposta. Os parâmetros precisão, exatidão e sensibilidade são adequados para o diagnóstico das intoxicações por estes compostos. O método foi aplicado em um estudo cinético de etanol com 6 voluntários, onde se observou uma gradativa redução da razão média de concentração de etanol entre fluido oral e sangue de 2,61 no tempo $0,5 \mathrm{~h}$ após a ingesta para 1,15 após $3 \mathrm{~h}$. Desta forma, não foi possível estimar com exatidão a concentração de etanol no sangue a partir das concentrações obtidas nas amostras de fluido oral.

Tabela 2. Parâmetros de validação do método

\begin{tabular}{|c|c|c|c|c|c|c|c|c|c|c|c|}
\hline \multirow[b]{2}{*}{ Analito } & \multirow[b]{2}{*}{$\begin{array}{c}\text { Concentração } \\
(\mu \mathrm{g} / \mathrm{mL})\end{array}$} & \multicolumn{5}{|c|}{ Sangue } & \multicolumn{5}{|c|}{ Fluido Oral } \\
\hline & & $\begin{array}{c}\text { Precisão } \\
\text { intraensaios ir } \\
\text { (C.V. \%) }\end{array}$ & $\begin{array}{l}\text { Precisão } \\
\text { interensaios } \\
\text { (C.V. \%) }\end{array}$ & $\begin{array}{c}\text { Exatidão } \\
(\%)\end{array}$ & Equação & $\mathrm{r}^{2}$ & $\begin{array}{c}\text { Precisão } \\
\text { intraensaios } \\
\text { (C.V. \%) }\end{array}$ & $\begin{array}{c}\text { Precisão } \\
\text { interensaios } \\
\text { (C.V. \%) }\end{array}$ & $\begin{array}{l}\text { Exatidão } \\
(\%)\end{array}$ & Equação & $\mathrm{r}^{2}$ \\
\hline \multirow[t]{3}{*}{ Acetaldeído } & 4000 & 6,1 & 8,2 & 89,1 & \multirow{3}{*}{$\begin{array}{c}y=0,0001 x \\
-0,0062\end{array}$} & \multirow[t]{3}{*}{0.9979} & 4,2 & 4,7 & 107,5 & \multirow{3}{*}{$\begin{aligned} y & =0,0001 x \\
& +0,0003\end{aligned}$} & \multirow[t]{3}{*}{0,9999} \\
\hline & 400 & 7,1 & 5,4 & 100,5 & & & 5,3 & 5,5 & 101,6 & & \\
\hline & 100 & 2,8 & 2,2 & 108,3 & & & 4,2 & 4,8 & 110,4 & & \\
\hline \multirow[t]{3}{*}{ Acetona } & 4000 & 6,1 & 6,0 & 96,5 & \multirow{3}{*}{$\begin{aligned} y & =0,0004 x \\
& +0,0088\end{aligned}$} & \multirow[t]{3}{*}{0,9991} & 3,9 & 7,5 & 102,1 & \multirow{3}{*}{$\begin{array}{c}y=0,0004 x \\
-0,0046\end{array}$} & \multirow[t]{3}{*}{0,9999} \\
\hline & 400 & 7,8 & 6,3 & 95,8 & & & 4,2 & 4,2 & 104,5 & & \\
\hline & 100 & 2,8 & 2,2 & 91,3 & & & 3,9 & 2,6 & 103,4 & & \\
\hline \multirow[t]{3}{*}{ Etanol } & 4000 & 6,7 & 4,2 & 90,1 & \multirow{3}{*}{$\begin{array}{c}y=0,0003 x \\
-0,0027\end{array}$} & \multirow[t]{3}{*}{0,9999} & 3,7 & 5,8 & 91,6 & \multirow{3}{*}{$\begin{array}{c}y=0,0003 x \\
-0,0051\end{array}$} & \multirow[t]{3}{*}{0,9999} \\
\hline & 400 & 3,4 & 3,8 & 92,3 & & & 2,7 & 3,7 & 95,8 & & \\
\hline & 100 & 6,3 & 5,0 & 96,8 & & & 1,8 & 2,4 & 100,5 & & \\
\hline \multirow[t]{3}{*}{ Isopropanol } & 4000 & 4,2 & 4,1 & 89,8 & \multirow{3}{*}{$\begin{array}{c}y=0,0007 x \\
-0,0101\end{array}$} & \multirow[t]{3}{*}{0,9995} & 3,7 & 5,8 & 101,5 & \multirow{3}{*}{$\begin{array}{c}y=0,0006 x \\
-0,0113\end{array}$} & \multirow[t]{3}{*}{0,9995} \\
\hline & 400 & 8,0 & 5,6 & 90,9 & & & 2,7 & 3,7 & 104,1 & & \\
\hline & 100 & 8,5 & 4,5 & 101,3 & & & 1,8 & 2,4 & 109,0 & & \\
\hline \multirow[t]{3}{*}{ Metanol } & 4000 & 3,2 & 3,2 & 100,5 & \multirow{3}{*}{$\begin{aligned} y= & 0,00007 x \\
& +0,005\end{aligned}$} & \multirow[t]{3}{*}{0,9973} & 3,7 & 4,7 & 107,1 & \multirow{3}{*}{$\begin{array}{c}y=0,00008 x \\
-0,0002\end{array}$} & \multirow[t]{3}{*}{0,9999} \\
\hline & 400 & 3,0 & 3,3 & 107,1 & & & 3,8 & 3,5 & 102,3 & & \\
\hline & 100 & 7,2 & 7,9 & 108,2 & & & 3,2 & 2,7 & 103,9 & & \\
\hline
\end{tabular}


Tabela 3. Concentrações de etanol em sangue e fluido oral obtidas no estudo cinético com voluntários

\begin{tabular}{|c|c|c|c|c|}
\hline $\begin{array}{l}\text { Voluntário } \\
\text { (sexo) }\end{array}$ & $\begin{array}{c}\text { Tempo após ingesta } \\
\text { (h) }\end{array}$ & $\begin{array}{l}\text { Concentração etanol } \\
\text { sangue }(\mu \mathrm{g} / \mathrm{mL})\end{array}$ & $\begin{array}{l}\text { Concentração de } \\
\text { etanol fluido oral }(\mu \mathrm{g} / \mathrm{mL})\end{array}$ & $\begin{array}{l}\text { Razão concentração fluido } \\
\text { oral/ concentração sangue }\end{array}$ \\
\hline \multirow[t]{4}{*}{$1(\mathrm{~F})$} & 0,5 & 248,3 & 715,3 & 2,88 \\
\hline & 1,0 & 341,3 & 668,0 & 1,96 \\
\hline & 2,0 & 301,3 & 511,3 & 1,70 \\
\hline & 3,0 & 200,3 & 247,0 & 1,23 \\
\hline \multirow[t]{4}{*}{$2(\mathrm{~F})$} & 0,5 & 178,0 & 661,6 & 3,72 \\
\hline & 1,0 & 417,6 & 779,3 & 1,87 \\
\hline & 2,0 & 435,3 & 593,3 & 1,36 \\
\hline & 3,0 & 291,0 & 364,0 & 1,25 \\
\hline \multirow[t]{4}{*}{$3(\mathrm{~F})$} & 0,5 & 173,6 & 316,0 & 1,82 \\
\hline & 1,0 & 195,0 & 286,3 & 1,47 \\
\hline & 2,0 & 166,6 & 238,0 & 1,43 \\
\hline & 3,0 & 132,0 & 167,3 & 1,27 \\
\hline \multirow[t]{4}{*}{$4(\mathrm{~F})$} & 0,5 & 398,6 & 771,6 & 1,94 \\
\hline & 1,0 & 608,3 & 1204 & 1,98 \\
\hline & 2,0 & 635,3 & 650,3 & 1,02 \\
\hline & 3,0 & 518,3 & 569,3 & 1,10 \\
\hline \multirow[t]{4}{*}{$5(\mathrm{M})$} & 0,5 & 516,0 & 1383,6 & 2,68 \\
\hline & 1,0 & 642,3 & 1263,3 & 1,97 \\
\hline & 2,0 & 479,6 & 848,6 & 1,77 \\
\hline & 3,0 & 386,0 & 442,6 & 1,15 \\
\hline \multirow[t]{4}{*}{$6(\mathrm{M})$} & 0,5 & n.d. & 91,0 & - \\
\hline & 1,0 & 329,6 & 650,6 & 1,97 \\
\hline & 2,0 & 276,3 & 240,6 & 0,87 \\
\hline & 3,0 & 202,6 & 181,0 & 0,89 \\
\hline
\end{tabular}

n.d.: não detectado

\section{AGRADECIMENTOS}

À Greiner Bio-One, pelo fornecimento dos sistemas para coleta de fluido oral utilizados no estudo. Ao Centro Universitário Feevale, pelo apoio financeiro.

\section{REFERÊNCIAS}

1. http://www.denatran.gov.br/ultimas/20080626_alcool_direcao.htm, acessada em Fevereiro 2009.

2. Baselt, R.; Disposition of Toxic Drugs and Chemicals in Man, $6^{\text {th }}$ ed., Biomedical Publications: Foster City, 2002.

3. Kugelberg, F.; Jones, A. W.; Forensic Sci. Int. 2007, 165, 10.

4. Zuba, D.; Piekoszewski, W.; Pach, J.; Winnik, L.; Parczewski, A.; Alcohol 2002, 26, 17.

5. Crouch, D. J.; Forensic Sci. Int. 2007, 150, 165.

6. Machata, G. Z.; Rechtsmed. 1975, 75, 229.
7. Zuba, D.; Parczewski, A.; Reichenbächer, M. J.; Chromatogr., B: Anal. Technol. Biomed. Life Sci. 2002, 773, 75.

8. De Martinis, B. S.; Martin, C. C. S.; Forensic Sci. Int. 2002, 128, 115.

9. De Martinis, B. S.; Ruzzene, M. A. M.; Martin, C. C. S.; Anal. Chim. Acta 2004, 522, 163.

10. Myers, R. H.; Montgomery, D. C.; Response Surface Methodology, $2^{\text {nd }}$ ed., Wiley-Interscience: New York, 2002.

11. Derringer, G.; Suich, R.; J. Qual. Technol. 1980, 12, 214.

12. Wieling, J.; Hendriks, G.; Tamminga, W. J.; Hempenius, J.; Mensink, C. K.; Oosterhuis, B.; Jonkman, J. H. G.; J.Chromatogr., A 1996, 730, 381.

13. Shah, V. P.; Midha, K. K.; Findlay, J. W.; Hill, H. M.; Hulse, J. D.; McGilveray, I. J.; McKay, G.; Miller, K. J.; Patnaik, R. N.; Powell, M. L.; Tonelli, A; Viswanathan, C. T.; Yacobi, A.; Pharm. Res. 2000, 17, 1551.

14. Jones, A. W.; Clin. Chem. 1979, 25, 1394.

15. Jones, A. W.; Clin. Chem. 1993, 39, 1837.

16. Gubala, W.; Zuba, D.; Pol. J. Pharmacol. 2002, 54, 161. 